\title{
Red Blood Cell Membrane-Coated Silica Nanoparticles Codelivering DOX and ICG for Effective Lung Cancer Therapy
}

\author{
Jia Xiao, Jie Weng, Fang Wen, and Juan Ye*
}

Cite This: ACS Omega 2020, 5, 32861-32867

Read Online

ABSTRACT: The effective chemotherapy of cancer is usually hindered by the unsatisfied cell internalization of the drug delivery systems (DDS) as well as drug resistance of cancer cells. In order to solve these dilemmas in one design, red blood cell membrane (RBM)-coated silica nanoparticles (RS) were fabricated to codeliver doxorubicin (Dox) and indocyanine green (ICG) to effectively treat the model lung cancer using photothermal-assisted chemotherapy. Our results demonstrated that the RS/I-D was the nanoparticle at around $100 \mathrm{~nm}$ with superior stability and biocompatibility. Especially, the photothermal effects of ICG were well preserved and could be

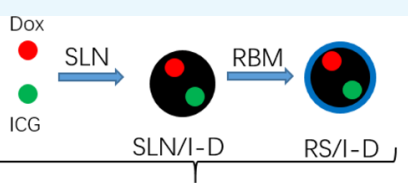

Photothermal assisted chemotherapy of drug resistance cancer applied to accelerate the drug release from the DDS. More importantly, the RBM modification can mediate enhanced cell internalization of drugs as compared to their free forms, which finally resulted in enhanced anticancer efficacy in Dox-resistant A549 cells (A549/Dox) both in vitro and in vivo with enhanced cell apoptosis and cell arrest.

\section{INTRODUCTION}

Recent studies in cancer therapy have revealed some reasons responsible for the poor performance of some drug delivery systems (DDS) in cancer therapy, among which unsatisfied cell internalization of the DDS as well as drug resistance of cancer cells are recognized as two important obstacles. ${ }^{1-3}$ It was well recognized that free drugs are usually subjected to quick excretion while nanoparticle-based DDS can increase the circulation time of loaded drug. ${ }^{4,5}$ However, because of their ectogenic nature, nanoparticles without careful surface modifications are prone to be captured by the reticuloendothelial system to trigger premature excretion. ${ }^{6,7}$ In order to solve this dilemma, polyethylene glycol (PEG) modification is becoming a widely recognized approach to increase the circulation time of the DDS, which has been verified by many previous studies. ${ }^{8-10}$ However, increasing evidences have revealed that although increasing the circulation time, the hydrophilic nature of PEG fails to satisfy decent cell internalization because of the opposite nature of cell membrane. As an alternative measure, the targeting moiety, which specifically recognizes the overexpressed receptors on the surface of cancer cells, was employed as a conjugation ligand for surface modification of DDS. Although some promising results were obtained, the complicated synthetic procedure as well as the inevitable cytotoxicity because of the residual side products severely hindered the further application of this method in cancer therapy. ${ }^{11-14}$

Until recently, the introduction of cell membrane-derived vehicles as the main component or accessory structures (such as the surface material) is becoming the best solution for the abovementioned dilemma. ${ }^{15-17}$ First, the cell membrane derivatives inherit the whole proteins of the mother cells, which show similar properties to the mother cells when being prepared into DDS. ${ }^{18}$ Most importantly, the same lipid bilayer structure between cell membrane derivatives and cancer cell membrane also makes it a suitable DDS for drug delivery with enhanced cell internalization. ${ }^{19}$ Finally, the cell membrane derivatives are of completely natural origin, with high biocompatibility and high accessibility. ${ }^{20}$ As a result, recent studies have devoted extensive efforts to explore the DDS potential of cell membrane derivatives. In particular, the cell membrane derived from red blood cell (RBC), which inherits the long circulation nature of $\mathrm{RBC}$, is acquiring more and more attentions in cancer therapy. ${ }^{21,22}$

In recent decades, DDS based on nanoparticles have showed many advantages over free drugs in drug delivery, such as elevated bioavailability and reduced side effects, which is widely recognized as an indispensable tool for cancer therapy. ${ }^{23,24}$ Therefore, various DDS have been developed and tested based on nanoparticles composed of either organic or inorganic materials. ${ }^{25-28}$ Silica nanoparticles (SLN), which have versatile virtues such as high biocompatibility, facile fabrication, and efficient nucleic acid binding, are becoming the suitable carrier for drug and especially gene delivery. Therefore, a number of DDS have been developed based on SLN, which showed satisfying outcomes. ${ }^{29-31}$

The chemotherapy of doxorubicin (Dox), a wide spectrum anticancer drug, was usually subjected to the acquired drug resistance of many cancers. ${ }^{32,33}$ As a result, alternative measures to employ additional aids to destroy tumors in a

Received: April 5, 2020

Accepted: July 16, 2020

Published: December 17, 2020 

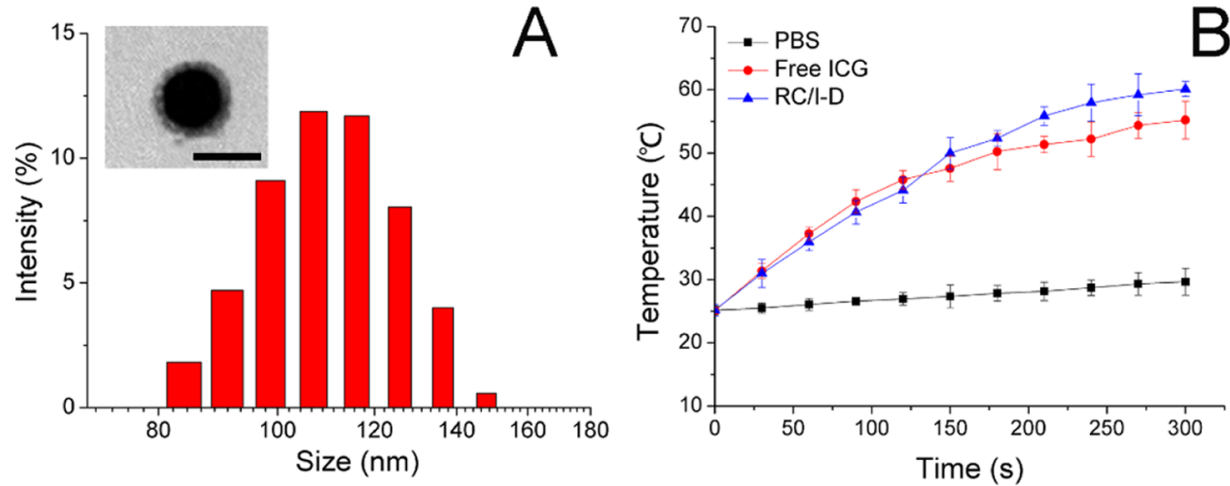

Figure 1. (A) Size distribution of RS/I-D. Inserted was the transmission electron microscopy image of RS/I-D. Scale bar is $100 \mathrm{~nm}$. (B) Photothermal capacity of RS/I-D in comparison to free ICG and PBS. Data were expressed as the mean standard deviation of three samples.
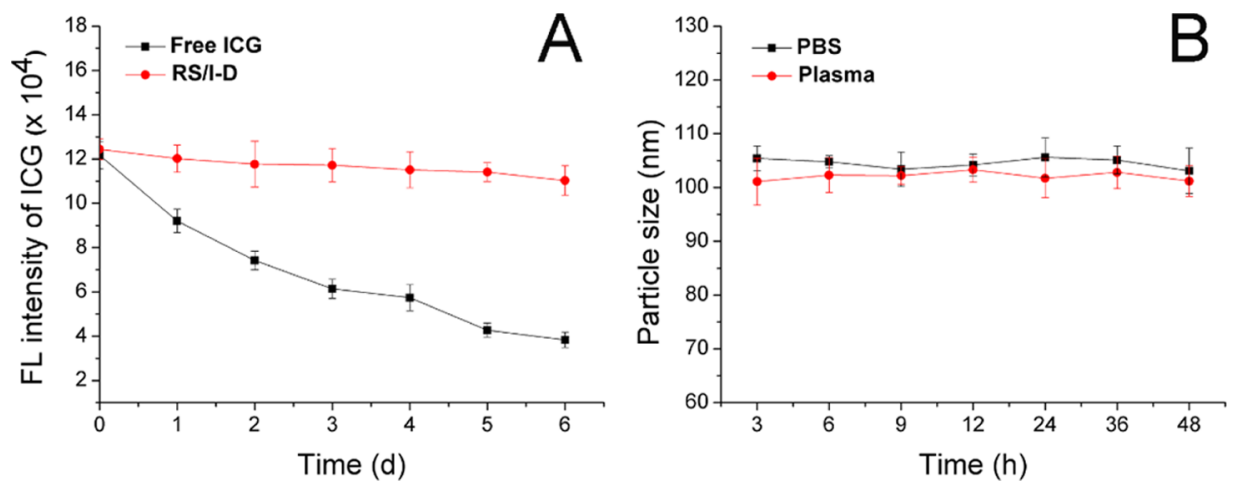

Figure 2. (A) Comparative fluorescence stability of free ICG and RS/I-D under sunlight for 6 days. (B) Colloidal stability of RS/I-D in PBS (pH 7.4) and mouse plasma at $37^{\circ} \mathrm{C}$ for up to $48 \mathrm{~h}$. Data were expressed as the mean standard deviation of three independently prepared nanoparticle preparations.

synergetic manner were well recognized as a feasible approach. ${ }^{34,35}$ As a result, in this study, size-controlled SLN was first synthesized, and Dox and indocyanine green (ICG) were loaded into the SLN during this process (SLN/I-D). After coating the SLN/I-D with the RBC membrane (RBM), the dual-loaded RBM-coated silica nanoparticles (RS)/I-D was prepared as a long circulation DDS for photothermal-assisted chemotherapy. It was suggested that surface modification of RBM can aid the RS/I-D to avoid premature excretion for enhanced tumor accumulation. Then, the intracellular photothermal nature of ICG achieves synergetic anticancer efficacy with Dox for effective therapy of Dox-resistant lung cancer (A549). The combination of RBM with SLN as the carrier and its potential in photothermal-assisted chemotherapy of drug resistance cancer is not fully explored by previous studies, which might be the core value of our study.

\section{RESULTS AND DISCUSSION}

2.1. Preparation of RS/I-D. To combine the advantages of high drug-loading capacity and biocompatibility in one DDS, SLN, as a widely adopted biomaterial, was used for the construction of drug-loaded core nanoparticles using the chemical precipitation method in the water-in-oil microemulsion. The Dox and ICG were preloaded into the matrix of SLN during synthesis (SLN/I-D). The RBM was finally coated onto SLN/I-D to finally prepare RS/I-D. As shown in Figure 1A, mean diameter determined by dynamic light scattering was around $110 \mathrm{~nm}$ with acceptable distribution, which was slightly larger than the size of SLN/I-D $(98.52 \mathrm{~nm}$, polydispersity index of 0.201 ), suggesting the successful coating of RBM. The coating of RBM was also supported by the changes of zeta potential (from $+25.23 \mathrm{mV}$ of SLN/I-D to $-12.16 \mathrm{mV}$ of RS/I-D, data not shown).

Afterward, the photothermal capacity of the loaded ICG within RS/I-D was further determined using $808 \mathrm{~nm}$ laser irradiation. As shown in Figure 1B, as compared to phosphatebuffered saline (PBS), the ICG-containing groups showed significant temperature rise upon the irradiation of $808 \mathrm{~nm}$. In particular, it was noted that free ICG showed lower final temperature as compared to that of RS/I-D. It was suggested that ICG was susceptible to the irradiation of light and prone to be degraded. As a result, it was suggested that the degradation of ICG at higher temperature might be responsible for the lower final temperature because a large proportion of ICG degraded without exerting their photothermal effects. In contrast, the RS/I-D might be able to protect the encapsulated ICG molecule to achieve satisfying photothermal effects.

2.2. Characterization of RS/I-D. ICG is a photosensitizer, which is vulnerable to the irradiation of lights. In order to further confirm the protective effect of RS on the ICG, the fluorescence intensity changes of RS/I-D in comparison to free ICG under the sunlight were further monitored. As shown in Figure 2A, the fluorescence intensity of free ICG suffered great decline upon the sunlight irradiation for merely 1 day and steadily decreased in a relatively rapid speed in the following days. In contrast, the fluorescence intensity of RS/I-D only decreased slightly after expose to sunlight. The total loss of fluorescence intensity was only $11.3 \%$ at Day 6 as compared to the $68.5 \%$ of that in the free ICG group. These results clearly demonstrated that RS could offer satisfactory protection to 

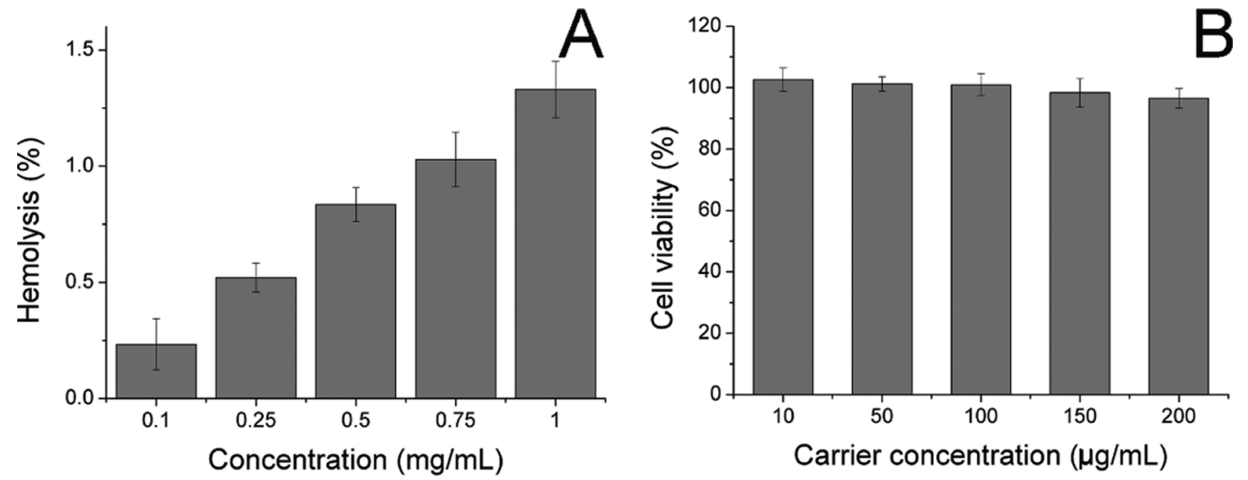

Figure 3. (A) Hemolysis of RS/I-D on $2 \%$ RBC under different concentrations at $37^{\circ} \mathrm{C}$ for $1 \mathrm{~h}$. (B) Cytotoxicity of various concentrations of drugfree carriers on A549/Dox cells for $48 \mathrm{~h}$. Data were expressed as the mean standard deviation of three samples.
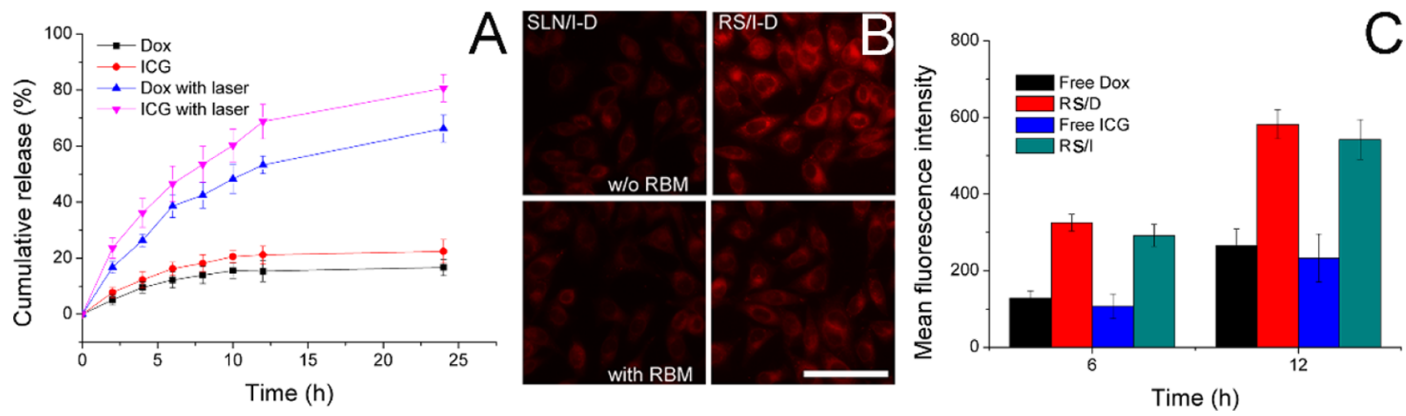

Figure 4. (A) Drug-release profiles of Dox and ICG from RS/I-D in PBS ( $\mathrm{pH} 7.4$ ) with or without laser irradiation ( $+\mathrm{L}$ means with light irradiation). (B) Intracellular fluorescence signal of Dox in A549/Dox cells incubated with SLN/I-D or RS/I-D with or without RBM pretreatment $(2 \mathrm{~h})$ for $4 \mathrm{~h}$. Scale bar: $100 \mu \mathrm{m}$. (C) Intracellular fluorescence signal of Dox and ICG at different time intervals incubated with free drugs (Dox and ICG) and corresponding DDSs (RS/D and RS/I) in A549/Dox cells using flow cytometry. Data were expressed as the mean standard deviation of three samples.

ICG to avoid the degradation of sunlight irradiation, which is beneficial for the safe delivery of sufficient drugs to the tumor tissue for better anticancer therapy.

Considering that the colloidal stability is a critical parameter to evaluate the performance of the DDS, the colloidal stability of RS/I-D under two physiological conditions (PBS 7.4 and mouse plasma) was therefore investigated. According to previous reports, the size of the DDS should maintain stability for a relatively long period to allow the safe delivery of loaded drug molecules to the target tissue without leakage. ${ }^{10,14,25}$ As a result, the particle size changes of RS/I-D were selected to be the indicator to reflect the colloidal stability. As shown in Figure 2B, during $48 \mathrm{~h}$ of incubation, the size of RS/I-D only showed minor variations in both PBS ( $\mathrm{pH} 7.4)$ and mouse plasma. Considering the instrumental error, it was therefore concluded that RS/I-D was a stable DDS under physiological conditions that might be suitable for cancer-related drug delivery.

Afterward, the biocompatibility of the carrier as well as RS/I$\mathrm{D}$ was studied. The hemolysis assay of RS/I-D was first investigated by incubating the DDS with $2 \% \mathrm{RBC}$ of $\mathrm{Balb} / \mathrm{c}$ mice to reflect the irritation of nanoparticles on $\mathrm{RBC}$ in the blood. As illustrated in Figure 3A, only 1.33\% hemolysis rate was obtained at the highest RS/I-D concentration of $1 \mathrm{mg} / \mathrm{mL}$. It was also well known that the actual DDS concentration upon in vivo application would be much lower than the threshold of $1 \mathrm{mg} / \mathrm{mL}$ because of the dilution body fluids (including blood and lymph). Therefore, the RS/I-D was concluded to be a safe DDS without significant risk of inducing hemolysis on RBC.
To further determine the cytotoxicity of drug-free carrier on cancer cells upon arrival of the target tissue, the drug-free carrier (RS) was incubated with A549/Dox cells at various concentrations for $48 \mathrm{~h}$, and the cell viability after treatment was studied. As displayed in Figure 3B, the cell viability of A549/Dox cells at $48 \mathrm{~h}$ postincubation remained still over $90 \%$ at the high concentration $(200 \mu \mathrm{g} / \mathrm{mL})$, indicating the potential of RBM-derived carrier to be a highly biocompatible carrier. Moreover, it was also suggested that the carrier showed almost no cytotoxicity effects on the cells, indicating that the results in the following assays were because of the effects of drugs but not the interference of carriers.

The DLC of Dox in RS/I-D was determined as $9.63 \%$ and the ICG was $9.54 \%$ using UV spectrophotometry.

2.3. Drug Release and Cellular Uptake. In order to understand the drug-release profile of RS/I-D under different conditions, the Dox and ICG release of RS/I-D $(5 \mathrm{mg} / \mathrm{mL})$ were evaluated with or without laser irradiation. As displayed in Figure 4A, under extracellular physiological condition ( $\mathrm{pH}$ 7.4), the drug release of both Dox and ICG was relatively slow with a final cumulative release percentage of 16.71 and $22.43 \%$, respectively, at $24 \mathrm{~h}$ postincubation. In contrast, upon laser irradiation, the release of both drugs was significantly elevated, indicating that the photothermal nature of ICG might facilitate the release of both drugs from the DDS. In detail, the total drug release percentage at the end of test $(24 \mathrm{~h})$ was 66.26 and $80.67 \%$, respectively. Therefore, it was inferred that RS/I-D was able to maintain stability at extracellular with minor drug leakage while transferred to a burst release state upon laser 

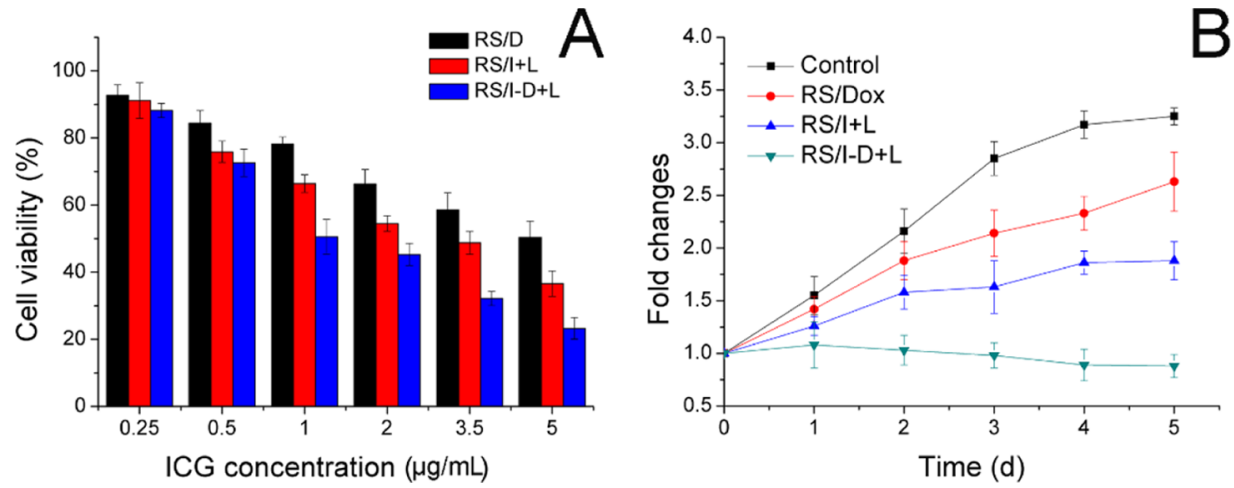

Figure 5. (A) Viability of A549/Dox cells treated with different formulations at different drug concentrations for 48 h. (B) Volume changes of MCTS after different treatments. Data were expressed as the mean standard deviation of three samples.
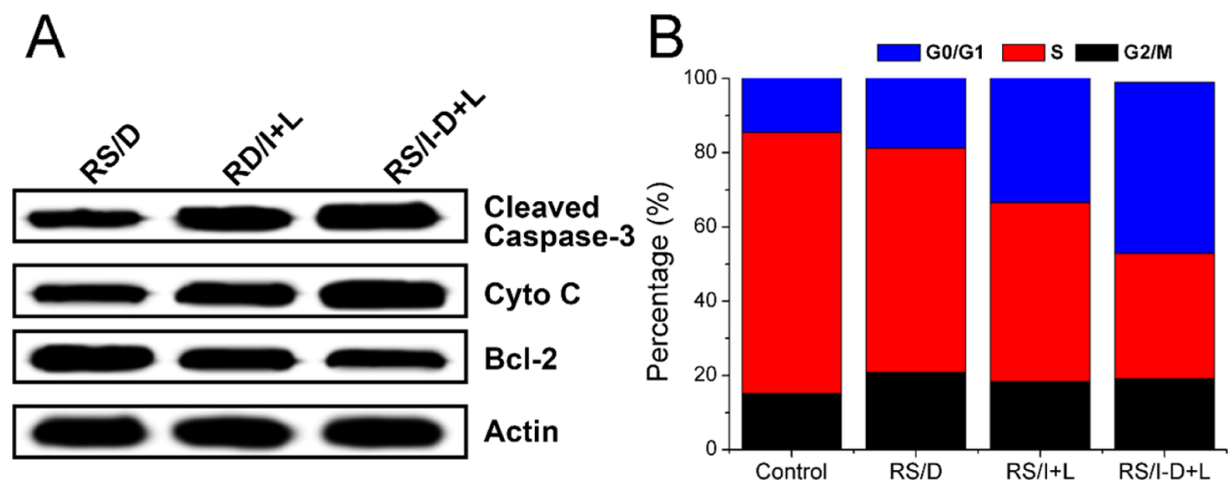

Figure 6. (A) Western blot assays of the expression of caspase-3, cytochrome $C$, and bcl-2 proteins after different treatments (drug dosage: $3.5 \mu \mathrm{g} /$ $\mathrm{mL}$ for $48 \mathrm{~h}$ ). (B) Cell cycle variations of A549/Dox cells treated with different formulations (drug dosage: $3.5 \mu \mathrm{g} / \mathrm{mL}$ for $48 \mathrm{~h}$ ).

irradiation, which was beneficial for realizing cancer-specific drug delivery for effective cancer therapy.

The cellular uptake of drugs incubated with or without RBM pretreatment was assessed. As shown in Figure 4B, compared with RBM-unmodified SLN/I-D, the cellular accumulation of RS/I-D at $4 \mathrm{~h}$ postincubation was much more elevated, suggesting the preferable cell internalization of RBM modification because of the similar nature between RBM and the cancer cell membrane. Interestingly, it was also confirmed by the competitive assay that pretreatment with RBM significantly reduced the cellular accumulation of RS/I-D while showed minor effects on SLN/I-D. As a result, we further concluded that the enhanced cell internalization was realized through the RBM-mediated cellular uptake.

Afterward, the comparative cellular uptake of drugs in their free forms (free Dox and free ICG) in comparison to their corresponding DDSs (RS/D and RS/I) was conducted. As shown in Figure 4C, after incubation with different groups for various time intervals ( 6 and $12 \mathrm{~h}$ ), it was observed that the intracellular accumulation of all groups was positively related to the incubation time. Moreover, because of the drug-resistant nature of A549/Dox, both free drugs were poorly accumulated within cells, and in particular, because of the hydrophilic nature of ICG, the ICG showed inferior accumulation to that of Dox. Most importantly, the drug accumulation in cells using corresponding DDSs was significantly enhanced, which was in line with previous reports that DDS can realize enhanced cell internalization of drugs than free drugs. ${ }^{36,37}$

2.4. In Vitro Anticancer Effect. The in vitro anticancer effect was conducted by classic MTT assay. The Dox and ICG within RS/I-D were around 1 and was adopted in this and the following assays. The results, as shown in Figure 5A, demonstrated that the anticancer effect of all formulations was positively related to the drug concentrations. Specifically, when Dox concentration reached $5 \mu \mathrm{g} / \mathrm{mL}$, the survival rate of A549/Dox cells in the RS/D group was still 50.4\%, suggesting the strong drug-resistant nature of this cell line. In contrast, RS/I at the same drug concentration subjected to laser irradiation achieved enhanced anticancer outcome, indicating the powerful anticancer capacity of photothermal therapy. Most importantly, the combination of Dox and ICG using photothermal-assisted chemotherapy showed the best performance on suppressing the growth of A549/Dox cells, which demonstrated the promising synergetic effects between these drugs. $^{38}$

The multicellular tumor spheroid (MCTS) mimicking the in vivo solid tumor was adopted to study the anticancer efficacy of various formulations. Figure $5 \mathrm{~B}$ shows that the volume of MCTS in the RS/D group remained increasing throughout the experiment, further indicating that the drug resistance of A549/Dox cells significantly decreased the cytotoxicity of Dox. RS/I using photothermal therapy exhibited enhanced suppression of tumor growth. Most importantly, the combination of Dox and ICG in RS/I-D exhibited significantly enhanced anticancer efficacy with a suppressed volume growth. $^{39}$

To further verify this conclusion, the apoptosis of related proteins (caspase-3, bcl-2, and cytochrome-3) in different formulations was investigated. Figure $6 \mathrm{~A}$ exhibits that RS/I-Dtreated cells had the highest expression level of cleaved caspase-3 while had the lowest expression of bcl-2 (responsible for suppressing apoptosis) among all groups, which further 


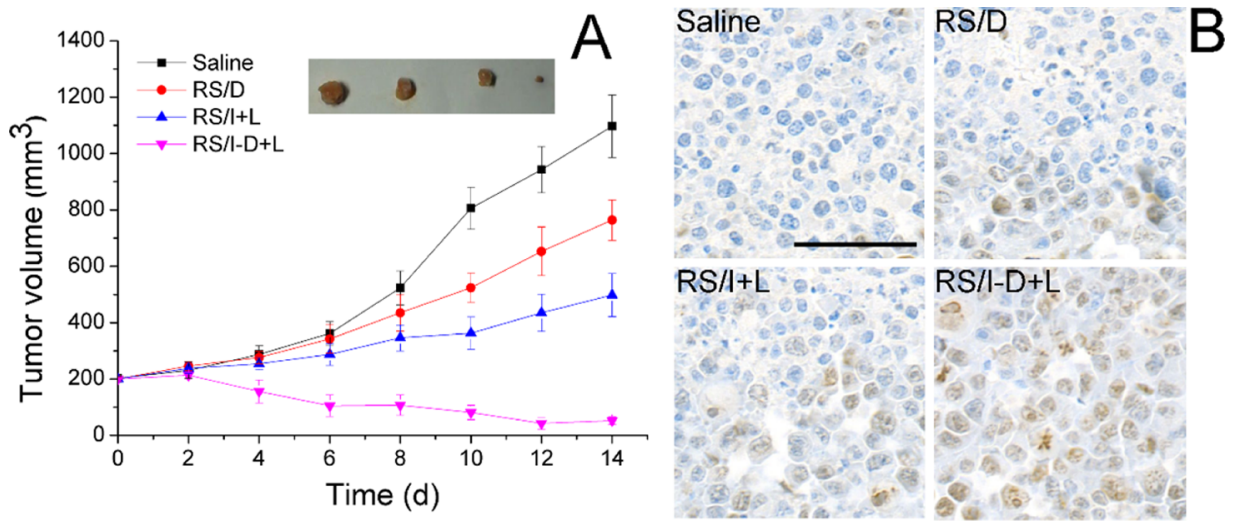

Figure 7. (A) Tumor volume variations of A549/Dox tumor-bearing Balb/c nude mice after administration of different formulations. Mice were intravenously administered with various formulations every other day for seven times, and each formulation contained the same dose of drugs (Dox and ICG: $5 \mathrm{mg} / \mathrm{kg}$ ). Inserted image was the representative tumor excised from the mice (from left to right: Saline, RS/D, RS/I + L, RS/I-D + L). Data were expressed as the mean standard deviation of six samples. (B) At the end of the test, the mice were sacrificed, and the tumor tissues from each group were subjected to TUNEL staining. Scale bar: $100 \mu \mathrm{m}$.

confirmed the superior anticancer effect of RS/I-D. Moreover, the RS/I-D exhibited much higher expression level of cytochrome-3, which indicated that mitochondria damage was also involved in the cell apoptosis. ${ }^{40}$

To illuminate the mechanism responsible for significantly increased cytotoxicity in the RS/I-D-treated group, the cell cycle variations after different treatments were studied. As shown in Figure 6B, compared with the control group, the cell percentage in the $S$ phase decreased from 70.2 to $60.4 \%$ in the $\mathrm{RS} / \mathrm{D}$ group and even to $48.2 \%$ in the RS/I group. In addition, the cell percentage in G0/G1 phase increased from 16.6 to $22.8 \%$ in the RS/D group and finally $33.4 \%$ in the RS/I group. In contrast, the $S$ phase and G0/G1 phase in the RS/I-D group was 33.7 and $44.1 \%$, respectively, which suggested much more severe cell cycle arrest in the RS/I-D group than the other groups.

2.5. In Vivo Anticancer Study. In vivo anticancer study of RS/I-D was performed. As displayed in Figure 6A, the saline group showed consistent growth of tumor tissue to a final volume of $1097 \pm 111 \mathrm{~mm}^{3}$. As expected, tumor growth was stunted to some extent upon administration with RS/D or RS/ I but still larger than the original volume without reversion. Nonetheless, the anticancer efficacy of mice in the RS/I-D group was much more potent than other groups with a significant reverse of tumor volume to $52 \pm 14 \mathrm{~mm}^{3}$. In addition, the tumor tissue from different groups was excised and subjected to TUNEL staining to analysis the apoptosis profiles after different treatments (Figure 7). As shown in Figure $6 \mathrm{~B}$, the tumor tissue from the RS/I-D group suffered from severe apoptosis then the other groups, which was characterized as the most widely observed positive cells (brown dots). ${ }^{41}$

\section{CONCLUSIONS}

In summary, we successfully developed a RBM-modified SLN to serve as a DDS for the codelivery of Dox and ICG (RS/I-D) for photothermal-assisted chemotherapy of drug-resistant A549/Dox cancer cells. The physicochemical characterizations showed that the RS/I-D exerted satisfactory distribution at around $100 \mathrm{~nm}$ with high stability, superior photothermal capacity, low hemolysis, and laser-responsive drug release. Cell experiments further demonstrated that RBM modification can mediate enhanced cellular uptake of RS/I-D into A549/Dox cells. Most importantly, the DDS can also enhance the cell internalization of free drugs, which achieved synergetic anticancer efficacy with elevated benefits than applying RS/D or RS/I alone. As expected, synergetic photothermal-assisted chemotherapy achieved much more elevated anticancer benefits than monotreatment means both in vitro and in vivo.

\section{EXPERIMENTAL SECTION}

4.1. Preparation of RS/I-D. The synthesis of ICG and Dox-loaded SLN (SLN/I-D) was achieved by using water-inoil microemulsion. In detail, the water-in-oil microemulsion $(10 \mathrm{~mL})$ containing Dox and ICG was prepared. Then, tetraethyl orthosilicate (5 mg), $\mathrm{N}$-(2-aminoethyl)-3-aminopropyltrimethoxysilane $(2 \mathrm{mg})$, and $\mathrm{NH}_{4} \mathrm{OH}(100 \mu \mathrm{L})$ were successively added into the microemulsion under vigorous agitation. After $24 \mathrm{~h}$ of reaction, SLN/I-D was precipitated by excess ethanol and collected by centrifugation (3000 rpm, 10 min, CR26, Hitachi, Japan).

In order to isolate RBM from RBCs, the RBCs were homogenized in $1 \mathrm{~mL}$ of extracting buffer (PBS, $0.0001 \mathrm{M}$ ) and further centrifuged $(10,000 \mathrm{~g}, 10 \mathrm{~min})$, followed by second ultracentrifugation $(100,000 \mathrm{~g}, 60 \mathrm{~min})$ to finally obtain the RBM. All procedures were performed at $4{ }^{\circ} \mathrm{C}$. The protein concentration of RBM was quantified using a BCA kit (Beyotime, Shanghai, China) according to manufacturer's instructions.

The RBM was then deposited onto the surface of SLN/I-D to construct RS/I-D. Briefly, $250 \mu \mathrm{L}$ of SLN/I-D $(1 \mathrm{mg} / \mathrm{mL})$ was mixed with RBM solution under vortex (w/w ratio of 5 ). Afterward, the mixture was subjected to probe-type sonication $(100 \mathrm{~W}, 5 \mathrm{~min})$. The mixture was further centrifuged $(10,000 \mathrm{~g}$, $10 \mathrm{~min}$ ) to collect RS/I-D.

Other detailed materials and methods can be found in the Supporting Information.

\section{ASSOCIATED CONTENT}

\section{Supporting Information}

The Supporting Information is available free of charge at https://pubs.acs.org/doi/10.1021/acsomega.0c01541.

The details for materials and methods (PDF) 


\section{AUTHOR INFORMATION}

\section{Corresponding Author}

Juan Ye - Department of Head and Neck Oncology, The Second Affiliated Hospital of Zunyi Medical University, Zunyi, Guizhou Province 563000, P. R. China; 이잉.org/ 0000-0002-1510-1248; Email: juanye777@163.com

\section{Authors}

Jia Xiao - Department of Clinical Oncology, The First People's Hospital of Yueyang, Yueyang, Hunan Province 414000, P. R. China

Jie Weng - Department of Clinical Oncology, The First People's Hospital of Yueyang, Yueyang, Hunan Province 414000, P. R. China

Fang Wen - Department of Clinical Oncology, The First People's Hospital of Yueyang, Yueyang, Hunan Province 414000, P. R. China

Complete contact information is available at:

https://pubs.acs.org/10.1021/acsomega.0c01541

\section{Notes}

The authors declare no competing financial interest.

\section{ACKNOWLEDGMENTS}

The authors acknowledge the language help from Letpub.

\section{REFERENCES}

(1) Kumari, P.; Rompicharla, S. V. K.; Bhatt, H.; Ghosh, B.; Biswas, S. Development of chlorin e6-conjugated poly(ethylene glycol)poly(d,l-lactide) nanoparticles for photodynamic therapy. Nanomedicine 2019, 14, 819-834.

(2) Tian, M.; Liu, C.; Dong, B.; Zuo, Y.; Lin, W. A dual-site controlled ratiometric probe revealing the simultaneous downregulation of $\mathrm{pH}$ in lysosomes and cytoplasm during autophagy. Chem. Commun. 2019, 55, 10440-10443.

(3) Zhao, X.; Tang, D. Y.; Zuo, X.; Zhang, T. D.; Wang, C. Identification of $\operatorname{lncRNA}-\mathrm{miRNA}-\mathrm{mRNA}$ regulatory network associated with epithelial ovarian cancer cisplatin-resistant. J. Cell. Physiol. 2019, 234, 19886-19894.

(4) Liu, Y.; Ibricevic, A.; Cohen, J. A.; Cohen, J. L.; Gunsten, S. P.; Fréchet, J. M. J.; Walter, M. J.; Welch, M. J.; Brody, S. L. Impact of Hydrogel Nanoparticle Size and Functionalization on In Vivo Behavior for Lung Imaging and Therapeutics. Mol. Pharm. 2009, 6, 1891-1902.

(5) He, H.; Guo, C.; Wang, J.; Korzun, W. J.; Wang, X.-Y.; Ghosh, S.; Yang, H. Leutusome: A biomimetic nanoplatform integrating plasma membrane components of leukocytes and tumor cells for remarkably enhanced solid tumor homing. Nano Lett. 2018, 18, 6164-6174.

(6) Chen, M.; Song, F.; Liu, Y.; Tian, J.; Liu, C.; Li, R.; Zhang, Q. A dual $\mathrm{pH}$-sensitive liposomal system with charge-reversal and $\mathrm{NO}$ generation for overcoming multidrug resistance in cancer. Nanoscale 2019, 11, 3814-3826.

(7) Kang, S.; Kang, K.; Chae, A.; Kim, Y.-K.; Jang, H.; Min, D.-H. Fucoidan-coated coral-like $\mathrm{Pt}$ nanoparticles for computed tomography-guided highly enhanced synergistic anticancer effect against drug-resistant breast cancer cells. Nanoscale 2019, 11, 15173-15183.

(8) Zhao, H.; Wang, Y.; Peng, J.; Zhang, L.; Qu, Y.; Chu, B.; Dong, M.; Tan, L.; Qian, Z. Biodegradable Self-Assembled Micelles Based on MPEG-PTMC Copolymers: An Ideal Drug Delivery System for Vincristine. J. Biomed. Nanotechnol. 2017, 13, 427-436.

(9) Wang, H.; Li, X.; Tse, B. W.-C.; Yang, H.; Thorling, C. A.; Liu, Y.; Touraud, M.; Chouane, J. B.; Liu, X.; Roberts, M. S.; Liang, X. Indocyanine green-incorporating nanoparticles for cancer theranostics. Theranostics 2018, 8, 1227.
(10) Duan, M.; Xia, F.; Li, T.; Shapter, J. G.; Yang, S.; Li, Y.; Gao, G.; Cui, D. Matrix metalloproteinase-2-targeted superparamagnetic $\mathrm{Fe}$ 3 O 4-PEG-G5-MMP2@ Ce6 nanoprobes for dual-mode imaging and photodynamic therapy. Nanoscale 2019, 11, 18426-18435.

(11) Wang, C.; Han, M.; Liu, X.; Chen, S.; Hu, F.; Sun, J.; Yuan, H. Mitoxantrone-preloaded water-responsive phospholipid-amorphous calcium carbonate hybrid nanoparticles for targeted and effective cancer therapy. Int. J. Nanomed. 2019, 14, 1503-1517.

(12) Zhang, Z.; Qian, H.; Huang, J.; Sha, H.; Zhang, H.; Yu, L.; Liu, B.; Hua, D.; Qian, X. Anti-EGFR-iRGD recombinant protein modified biomimetic nanoparticles loaded with gambogic acid to enhance targeting and antitumor ability in colorectal cancer treatment. Int. J. Nanomed. 2018, 13, 4961-4975.

(13) Sun, S.; Yunxue, X.; Peng, F.; Min, C.; Suhui, S.; Ranran, Z.; Jinrui, W.; Xiaolong, L.; Shumin, W. Ultrasound-targeted photodynamic and gene dual therapy for effectively inhibiting triple negative breast cancer by cationic porphyrin lipid microbubbles loaded with HIF $1 \alpha$-siRNA. Nanoscale 2018, 10, 19945-19956.

(14) Zhang, Y.; Cheng, M.; Cao, J.; Zhang, Y.; Yuan, Z.; Wu, Q.; Wang, W. Multivalent nanoparticles for personalized theranostics based on tumor receptor distribution behavior. Nanoscale 2019, 11, $5005-5013$

(15) Zhang, J.; Miao, Y.; Ni, W.; Xiao, H.; Zhang, J. Cancer cell membrane coated silica nanoparticles loaded with ICG for tumour specific photothermal therapy of osteosarcoma. Artif. Cells, Nanomed., Biotechnol. 2019, 47, 2298-2305.

(16) Rahimi, M.; Shafiei-Irannejad, V.; Safa, K. D.; Salehi, R. Multibranched ionic liquid-chitosan as a smart and biocompatible nanovehicle for combination chemotherapy with stealth and targeted properties. Carbohydr. Polym. 2018, 196, 299-312.

(17) Yang, J.; Teng, Y.; Fu, Y.; Zhang, C. Chlorins e6 loaded silica nanoparticles coated with gastric cancer cell membrane for tumor specific photodynamic therapy of gastric cancer. Int. J. Nanomed. 2019, 14, 5061 .

(18) Tehrani, R. M.; Verdi, J.; Noureddini, M.; Salehi, R.; Salarinia, R.; Mosalaei, M.; Simonian, M.; Alani, B.; Ghiasi, M. R.; Jaafari, M. R. Mesenchymal stem cells: A new platform for targeting suicide genes in cancer. J. Cell. Physiol. 2018, 233, 3831-3845.

(19) Wang, P.; Wang, X.; Luo, Q.; Li, Y.; Lin, X.; Fan, L.; Zhang, Y.; Liu, J.; Liu, X. Fabrication of red blood cell-based multimodal theranostic probes for second near-infrared window fluorescence imaging-guided tumor surgery and photodynamic therapy. Theranostics 2019, 9, 369.

(20) Enderami, S. E.; Soleimani, M.; Mortazavi, Y.; Nadri, S.; Salimi, A. Generation of insulin-producing cells from human adipose-derived mesenchymal stem cells on PVA scaffold by optimized differentiation protocol. J. Cell. Physiol. 2018, 233, 4327-4337.

(21) Dumitru, A. C.; Poncin, M. A.; Conrard, L.; Dufrêne, Y. F.; Tyteca, D.; Alsteens, D. Nanoscale membrane architecture of healthy and pathological red blood cells. Nanoscale Horiz. 2018, 3, 293-304.

(22) Jin, G.; He, R.; Liu, Q.; Lin, M.; Dong, Y.; Li, K.; Tang, B. Z.; Liu, B.; Xu, F. Near-infrared light-regulated cancer theranostic nanoplatform based on aggregation-induced emission luminogen encapsulated upconversion nanoparticles. Theranostics 2019, 9, 246.

(23) Huang, J.; Liu, F.; Han, X.; Zhang, L.; Hu, Z.; Jiang, Q.; Wang, Z.; Ran, H.; Wang, D.; Li, P. Nanosonosensitizers for highly efficient sonodynamic cancer theranostics. Theranostics 2018, 8, 6178.

(24) Wang, C.; Yu, F.; Liu, X.; Chen, S.; Wu, R.; Zhao, R.; Hu, F.; Yuan, H. Cancer-Specific Therapy by Artificial Modulation of Intracellular Calcium Concentration. Adv. Healthcare Mater. 2019, 8,1900501

(25) Zhao, X.; Tang, D.; Yang, T.; Wang, C. Facile preparation of biocompatible nanostructured lipid carrier with ultra-small size as a tumor-penetration delivery system. Colloids Surf., B 2018, 170, 355363.

(26) Tang, D.; Zhao, X.; Yang, T.; Wang, C. Paclitaxel prodrug based mixed micelles for tumor-targeted chemotherapy. RSC Adv. 2018, 8, 380-389. 
(27) Cong, V. T.; Ly, N. H.; Son, S. J.; Min, J.; Joo, S.-W. Silicaencapsulated gold nanoparticle dimers for organelle-targeted cellular delivery. Chem. Commun. 2017, 53, 5009-5012.

(28) Wu, H.; Zhao, Y.; Mu, X.; Wu, H.; Chen, L.; Liu, W.; Mu, Y.; Liu, J.; Wei, X. A silica-polymer composite nano system for tumortargeted imaging and p53 gene therapy of lung cancer. J. Biomater. Sci., Polym. Ed. 2015, 26, 384-400.

(29) Meng, L.-x.; Ren, Q.; Meng, Q.; Zheng, Y.-x.; He, M.-1.; Sun, S.y.; Ding, Z.-j.; Li, B.-c.; Wang, H.-y. Trastuzumab modified silica nanoparticles loaded with doxorubicin for targeted and synergic therapy of breast cancer. Artif. Cells, Nanomed., Biotechnol. 2018, 46, S556-S563.

(30) Chai, S.; Kan, S.; Sun, R.; Zhou, R.; Sun, Y.; Chen, W.; Yu, B. Fabricating polydopamine-coated MoSe2-wrapped hollow mesoporous silica nanoplatform for controlled drug release and chemophotothermal therapy. Int. J. Nanomed. 2018, 13, 7607-7621.

(31) Tambe, P.; Kumar, P.; Paknikar, K. M.; Gajbhiye, V. Decapeptide functionalized targeted mesoporous silica nanoparticles with doxorubicin exhibit enhanced apoptotic effect in breast and prostate cancer cells. Int. J. Nanomed. 2018, 13, 7669-7680.

(32) Da Silva, C. G.; Camps, M. G. M.; Li, T. M. W. Y.; Zerrillo, L.; Löwik, C. W.; Ossendorp, F.; Cruz, L. J. Effective chemoimmunotherapy by co-delivery of doxorubicin and immune adjuvants in biodegradable nanoparticles. Theranostics 2019, 9, 6485.

(33) Wang, C.; Wang, Z.; Zhao, X.; Yu, F.; Quan, Y.; Cheng, Y.; Yuan, H. DOX Loaded Aggregation-induced Emission Active Polymeric Nanoparticles as a Fluorescence Resonance Energy Transfer Traceable Drug Delivery System for Self-indicating Cancer Therapy. Acta Biomater. 2019, 85, 218-228.

(34) Huang, W.; Lang, Y.; Hakeem, A.; Lei, Y.; Gan, L.; Yang, X. Surfactin-based nanoparticles loaded with doxorubicin to overcome multidrug resistance in cancers. Int. J. Nanomed. 2018, 13, 1723.

(35) Singh, S. Liposome encapsulation of doxorubicin and celecoxib in combination inhibits progression of human skin cancer cells. Int. J. Nanomed. 2018, 13, 11.

(36) Tang, Y.; Li, Y.; Li, S.; Hu, H.; Wu, Y.; Xiao, C.; Chu, Z.; Li, Z.; Yang, $\mathrm{X}$. Transformable nanotherapeutics enabled by ICG: towards enhanced tumor penetration under NIR light irradiation. Nanoscale 2019, 11, 6217-6227.

(37) Xie, X.; Shao, X.; Ma, W.; Zhao, D.; Shi, S.; Li, Q.; Lin, Y. Overcoming drug-resistant lung cancer by paclitaxel loaded tetrahedral DNA nanostructures. Nanoscale 2018, 10, 5457-5465.

(38) Zhou, B.-G.; Wei, C.-S.; Zhang, S.; Zhang, Z.; Gao, H.-m. Matrine reversed multidrug resistance of breast cancer MCF-7/ADR cells through PI3K/AKT signaling pathway. J. Cell. Biochem. 2018, 119, 3885-3891.

(39) Zhang, X.; Li, Y.; Wei, M.; Liu, C.; Yu, T.; Yang, J. Cetuximabmodified silica nanoparticle loaded with ICG for tumor-targeted combinational therapy of breast cancer. Drug Delivery 2019, 26, 129136.

(40) Igarashi, K.; Li, S.; Han, Q.; Tan, Y.; Kawaguchi, K.; Murakami, T.; Kiyuna, T.; Miyake, K.; Li, Y.; Nelson, S. D.; Dry, S. M.; Singh, A. S.; Elliott, I. A.; Russell, T. A.; Eckardt, M. A.; Yamamoto, N.; Hayashi, K.; Kimura, H.; Miwa, S.; Tsuchiya, H.; Eilber, F. C.; Hoffman, R. M. Growth of doxorubicin-resistant undifferentiated spindle-cell sarcoma PDOX is arrested by metabolic targeting with recombinant methioninase. J. Cell. Biochem. 2018, 119, 3537-3544.

(41) Zhao, X.; Tang, D.; Wu, Y.; Chen, S.; Wang, C. An artificial cell system for biocompatible gene delivery in cancer therapy. Nanoscale 2020, 12, 10189-10195. 